\title{
Phosphorylation of the NBDY microprotein promotes dissociation of biomolecular condensates
}

\author{
Zhenkun $\mathrm{Na}^{125}$, Yang Luo ${ }^{125}$, Danica S. Cui ${ }^{1}$, Alexandra Khitun ${ }^{12}$, Stephanie Smelyansky ${ }^{123}$, J. \\ Patrick Loria ${ }^{13}$, Sarah A. Slavoff ${ }^{1234}$ \\ ${ }^{1}$ Department of Chemistry, Yale University, New Haven, Connecticut 06520, United States \\ ${ }^{2}$ Institute of Biomolecular Design and Discovery, Yale University \\ ${ }^{3}$ Department of Molecular Biophysics and Biochemistry, Yale University, New Haven, Connecticut 06529, United \\ States \\ ${ }^{4}$ sarah.slavoff@yale.edu \\ ${ }^{5}$ Equal contribution
}

\begin{abstract}
Proteogenomic identification of translated small open reading frames in human has revealed thousands of microproteins, or polypeptides of fewer than 100 amino acids, that were previously invisible to geneticists. Hundreds of microproteins have been shown to be essential for cell growth and proliferation, and many regulate macromolecular complexes, but the vast majority remain functionally uncharacterized, lack secondary structure, and exhibit limited evolutionary conservation. One such intrinsically disordered microprotein is NBDY, a 68-amino acid component of membraneless organelles known as P-bodies. In this work, we show that NBDY can undergo liquid-liquid phase separation, a biophysical process thought to underlie the formation of membraneless organelles, in the presence of RNA in vitro. Phosphorylation of NBDY drives liquid phase remixing in vitro and macroscopic P-body dissociation in cells undergoing growth factor signaling and cell division. These results suggest that NBDY phosphorylation is a key regulator of P-body dynamics in cells, and more broadly that intrinsically disordered microproteins may contribute to liquid-liquid phase separation and remixing behavior.
\end{abstract}

\section{Introduction:}

Small open reading frames (smORFs) encoding polypeptides of fewer than 100 amino acids have been largely excluded from genome annotations because they are challenging to differentiate from randomly-occurring, non-translated ORFs ${ }^{1}$. Recent advances in ribosome profiling and proteogenomics have enabled the discovery of thousands of expressed smORFs in the human genome ${ }^{2}$. A number of "microproteins" encoded in smORFs (also referred to as small proteins or micropeptides ${ }^{3}$ ) have now been shown to have important cellular and biological functions ${ }^{4}$, but the vast majority remain uncharacterized. Indeed, whether microproteins are broadly functional - and how to even define the term "function" in the context of smORFs ${ }^{5}$ - remains in question. Multiple studies suggest that smORFs represent "proto-genes", or instances of de novo gene birth, which have not yet been optimized for functionality by natural selection ${ }^{6-7}$. Supporting the proto-gene model, the products of short, evolutionarily young genes exhibit limited sequence homology to known protein domains ${ }^{8}$, and are enriched for amino acid compositions consistent with predicted intrinsic disorder'.

Intrinsically disordered protein regions can undergo weak, multivalent interactions with biomacromolecules that drive liquid-liquid phase separation (LLPS) to form membraneless organelles. We therefore propose that intrinsic disorder could be a feature, rather than a flaw, of microproteins, positioning them as a class of molecules that may collectively function in LLPS in cells. Supporting this hypothesis, several smORF-encoded microproteins have recently been shown to localize to the nucleolus ${ }^{10}$, cytoplasmic membraneless organelles ${ }^{11-12}$, and ER membrane contact sites ${ }^{13-14}$, all of which may represent phase separated regions ${ }^{15-17}$.

The NoBody (NBDY) microprotein localizes to cytoplasmic membraneless organelles called P-bodies via interaction with components of the mRNA decapping complex, which catalyzes the first committed step in 5'-to-3' decay of deadenylated $\mathrm{mRNA}^{11-12}$. P-bodies are conserved in all eukaryotes, and have been reported to exhibit properties of phaseseparated liquid droplets ${ }^{17}$. While P-bodies are not necessary for $5^{\prime}$-to- $3^{\prime}$ mRNA decay ${ }^{18}$, which can occur in the 
cytoplasm $^{19-20}$, recent evidence suggests that the activity of the mRNA decapping enzyme DCP2 is exquisitely controlled, both positively and negatively, by conformational changes and protein-protein interactions in the liquid droplet phase ${ }^{21}$.

Inspired by the foundational demonstration that positively charged peptides can form liquid droplets in the presence of RNA in vitro, and that phosphorylation of these peptide-RNA coacervates drives liquid phase remixing ${ }^{22}$, we propose that modification of NBDY could control phase separation of P-body components, as has been previously shown for several Pbody proteins whose phosphorylation promotes P-body dissociation ${ }^{23-24}$. We demonstrate that NBDY phase separates in the presence of RNA in vitro, and that phosphorylation of NBDY dissociates these liquid droplets. We further show that NBDY is a master regulator of P-bodies in cells, taking phosphorylation inputs from multiple signaling pathways to promote P-body disappearance prior to cell division. These results demonstrate that phosphorylation switches the positively charged, intrinsically disordered microprotein NBDY from promoting to preventing LLPS both in vitro and in cells, and suggest that microproteins could more generally contribute to formation and regulation of liquid condensates in cells.

Results:

\section{NBDY undergoes liquid-liquid phase separation in the presence of RNA in vitro}

NBDY bears a predicted charge of +4 at physiological $\mathrm{pH}$, with high local positive charge near its middle segment (residues 22-27). NBDY also contains proline-rich sequences and is predicted to lack stable secondary structure ${ }^{25-26}$. Because LLPS is associated with intrinsically disordered proteins ${ }^{25,27}$, and can occur via complex coacervation (nonspecific association of oppositely charged polyanions), we hypothesized that NBDY may undergo liquid-liquid phase separation (LLPS) in the presence of RNA - and that this process could be disrupted by phosphorylation ${ }^{22,28-31}$. First, to experimentally examine intrinsic disorder in NBDY, we performed a ${ }^{1} \mathrm{H}_{-}{ }^{15} \mathrm{~N}$ HSQC NMR experiment with backbonelabeled NBDY. The narrow distribution of NBDY cross-peaks indicated the absence of a well-defined three-dimensional structure in solution (Figure $\mathrm{S} 1)^{32}$.

We subsequently investigated the ability of NBDY to form liquid droplets in the presence of RNA in vitro. Neither NBDY nor an unstructured polyuridine polymer (poly(U)) form liquid droplets alone in solution (Figure 1A), but when mixed, NBDY and poly(U) RNA undergo LLPS (Figure 1A-B and Movie S1). Total RNA purified from HEK 293T promoted LLPS of NBDY in vitro as well (Figure 1C). NBDY phase separation requires co-partitioning with RNA, as indicated by co-localization of fluorescently labeled poly(U) RNA and NBDY in liquid droplets (Figure 1D). Liquid droplet formation measured as an increase in turbidity depends on the concentration of NBDY protein, as expected, and LLPS was maximal at NBDY concentrations above $200 \mu \mathrm{M}$ (Figure 1E and Figure S2A). Addition of $100 \mathrm{mM} \mathrm{NaCl}$ decreased the turbidity to $\sim 35 \%$, and the coacervates dissolved at $200 \mathrm{mM} \mathrm{NaCl}$, consistent with a requirement for chargecharge interactions (Figure 1F and Figure S2B). Taken together, these results are consistent with phase separation of NBDY in the presence of RNA via the electrostatic process of complex coacervation.

\section{NBDY phosphorylation is required for P-body disappearance during mitosis}

Cellular P-bodies contain hundreds of proteins and thousands of RNAs ${ }^{33}$, and thus are far more complex than the reductionist NBDY-RNA system that we examined in vitro. Nonetheless, we hypothesized that NBDY may contribute to, and regulate, P-body formation and maintenance in cells. In particular, we hypothesized that phosphorylation of NBDY could perturb local charge density to drive P-body dissociation. P-bodies dissociate during mitosis, when DCP1A and other P-body proteins are phosphorylated ${ }^{23,34}$. We therefore examined NBDY phosphorylation during mitosis. Phosphoproteomic analysis of NBDY immunopurified from cells treated with the G2/M checkpoint inhibitor nocodazole revealed phosphorylation at residue T40 via phosphoproteomic analysis (Figure 2A-B). We generated a NBDY T40 phospho-specific antibody, the specificity of which was demonstrated as specific binding to NBDY, but not a nonphosphorylatable point mutant of NBDY, both of which were isolated from nocodazole-treated cells; low binding to NBDY in the absence of nocodazole treatment; and low binding to phosphatase-treated NBDY isolated from nocodazoletreated cells (Figure 2C). We then quantified NBDY phosphorylation using Phos-tag SDS-PAGE followed by Western blotting with NBDY and NBDY T40 phospho-specific antibodies (Figure 2D). Under these conditions, NBDY phosphorylation at T40 reaches 50\% occupancy (Figure 2D). We next defined the signaling pathway upstream of NBDY phosphorylation at T40. Since cyclin-dependent kinases (CDKs) regulate the G2/M transition ${ }^{35}$, we hypothesized that NBDY would be phosphorylated by, or downstream of, CDK activity. Consistent with this hypothesis, the CDK-selective inhibitor AT7519 ${ }^{36}$ specifically prevented NBDY phosphorylation under nocodazole treatment, while the control compound wortmannin, an inhibitor of phosphoinositide 3-kinases (PI3K), did not (Figure 2E). NBDY is therefore phosphorylated at a single site at the G2/M checkpoint downstream of CDK activity.

Given the prior observation that single site phosphorylation of model peptides bearing four arginine residues was sufficient to promote liquid phase remixing of peptide-RNA coacervates ${ }^{22}$, we hypothesized that the G2/M checkpoint NBDY phosphorylation site could perturb P-body phase separation. To test the involvement of NBDY pT40 in mitotic Pbody loss, we stably expressed the nonphosphorylatable mutant NBDY T40A in a NBDY CRISPR/Cas9 knockout (KO) HEK 293 T cell line ${ }^{37}$ and compared P-body dynamics in these cells to cells expressing WT NBDY - which can be phosphorylated - on the same KO background (Figure S3A). Remarkably, while WT NBDY-expressing cells recapitulated 
previously reported mitotic P-body disassembly after $12 \mathrm{~h}$ nocodazole treatment, the T40A mutant-expressing cells exhibited no detectable decrease in P-bodies under the same conditions (Figure 3). Phosphorylation of NBDY at T40 by CDKs at the mitotic checkpoint is therefore necessary for P-body disappearance.

\section{NBDY phosphorylation is required for P-body disappearance during epidermal growth factor receptor (EGFR) signaling}

Because NBDY phosphorylation occurs during cell division, we reasoned that growth factor signaling could also lead to NBDY modification and P-body loss. Treatment of HEK 293T cells with epidermal growth factor (EGF) led to phosphorylation of NBDY at S61, as detected by phosphoproteomic analysis of immunopurified NBDY (Figure 4A-B). Resolution with Phos-Tag SDS-PAGE and immunoblotting with a NBDY S61 phospho-specific antibody of validated specificity showed $\sim 65 \%$ NBDY phosphorylation stoichiometry in the presence of EGF (Figure 4C-D). NBDY phosphorylation appeared within 5 min of EGF treatment, consistent with EGFR activation ${ }^{38}$ (Figure S3B), and as little as $1 \mathrm{ng} / \mathrm{ml}$ EGF induced detectable NBDY pS61 (Figure S3C). In order to further exclude confounding effects from supraphysiologic EGF concentrations, we hypothesized that cancer-associated activating mutations in EGFR could increase endogenous NBDY pS61 without the need for growth factor addition. We therefore compared four non-small cell lung carcinoma lines: one expressing wild-type EGFR (A549), two with different activating EGFR mutations (HCC827 and H3255), and one activating mutation in EGFR combined with T790M conferring first-generation EGFR inhibitor resistance $(\mathrm{H} 1975)^{39}$. We found that the level of NBDY pS61 correlated with activation of EGFR without significantly changing NBDY expression (Figure 4E). Importantly, treatment of these cells with erlotinib, a first-generation EGFR inhibitor, abrogated NBDY pS61 in all cells except resistant H1975, confirming that NBDY phosphorylation requires EGFR signaling.

We then determined the kinase responsible for NBDY phosphorylation at S61. EGFR signals through a complex network of intermediates including ERK (extracellular signal-regulated kinase), MAPK (mitogen-activated protein kinase), PI3K (phosphoinositide 3-kinase)-AKT (protein kinase B), and PLC 11 (phospholipase C gamma 1)-PKC (protein kinase C $)^{35,36}$. Inhibitors of the ERK/MAPK and PI3K-AKT pathways, either alone or in combination, had no effect on EGF-dependent NBDY pS61 (Figure S4), while inhibition of PKC with bisindolylmaleimide II decreased EGF-dependent pS61 to the same extent as EGFR inhibition with AG1478 (Figure 4F). Purified PKC $\alpha$ can furthermore phosphorylate NBDY at S61 in vitro (Figure 4G), while control kinases cannot. It is therefore possible that PKC phosphorylates NBDY at S61 downstream of EGFR via a previously described PLC $\gamma$ pathway ${ }^{40-41}$.

We hypothesized that phosphorylation of NBDY during EGFR signaling could also drive P-body dissociation, but, unlike mitosis, the effect of EGF on P-bodies had not been previously reported. Remarkably, we found that EGF treatment at both $20 \mathrm{ng} / \mathrm{mL}$ and $100 \mathrm{ng} / \mathrm{mL}$ led to near complete P-body loss in wild-type HEK 293T over 30 min (Figure 5A-C). Expression of the nonphosphorylatable NBDY S61A mutant in the NBDY KO background abrogated EGF-dependent Pbody disappearance, while the phosphomimetic NBDY S61D mutant supported EGF-dependent P-body loss identical to expression of the WT NBDY coding sequence (Figure 6A-B). EGF-dependent P-body loss is not observed in NBDY KO cells, similar to the S61A mutant (Figure S5A). These results are consistent with a requirement of NBDY S61 phosphorylation for P-body loss during EGFR signaling. NBDY pS61 is necessary but not sufficient for P-body disappearance, because NBDY S61D rescue cells exhibit wild-type P-body numbers in the absence of EGF treatment (Figure 6B). Interactions of the decapping complex components DCP1A, EDC4 and NBDY are not perturbed by EGF treatment (Figure S5B), suggesting that NBDY phosphorylation is required for dissociation of P-bodies but does not disrupt individual decapping complexes. We therefore propose that phosphorylated NBDY is required for dissociation of P-bodies into sub-microscopic decapping complexes during both mitosis and EGF signaling, but altering the charge on NBDY in the absence of EGFR signaling is not sufficient for this effect. Rather, we propose that NBDY phosphorylation tips the balance of electrostatic repulsion within a network of P-body proteins that are also phosphorylated downstream of EGFR activation ${ }^{34}$.

\section{NBDY phosphorylation drives dissociation of NBDY/RNA coacervates in vitro}

To provide further support for our electrostatic model of P-body dissociation in a simple model system, we examined whether NBDY phosphorylation could dissociate NBDY-RNA coacervates in vitro. While this system lacks the complex network of P-body components, it is analogous to a previously reported phosphorylation-switchable peptide-RNA coacervate system ${ }^{22}$, and reports on whether alteration of the overall NBDY charge by phosphorylation is sufficient to disrupt electrostatic interactions with RNA. Since PKC phosphorylates NBDY at S61 downstream of EGFR and in vitro, we used PKC to achieve NBDY phosphorylation in coacervates. Addition of PKC to the in vitro NBDY-RNA LLPS system dissociates liquid droplets in a time-dependent manner (Figure 7A and Movie S2). As previously reported ${ }^{22}$, mixing fluorescently labeled NBDY, RNA and PKC in buffer containing PKC reaction components and ATP initially led to formation of liquid droplets that fell to the coverslip surface by 5 minutes, followed by remixing of the liquid phase that was essentially complete by 30 minutes. Similar results were obtained with unlabeled NBDY using turbidity measurements rather than fluorescence microscopy (Figure 7B, red trace). Importantly, purified NBDY S61A, the nonphosphorylatable mutant, formed liquid droplets in the presence of RNA under the PKC reaction conditions, but turbidity 
remained high in the presence of PKC (Figure 7B, blue trace), indicating that these liquid droplets were refractory to kinase activity. NBDY phosphorylation at S61 is therefore sufficient to promote liquid phase remixing in vitro, likely as a result of decreased overall or local positive charge.

\section{Discussion}

Intrinsically disordered, post-translationally modified microproteins have the potential to promote and dynamically regulate liquid phase-separated membraneless organelle formation. In this work, we demonstrated this principle in the context of the well-characterized microprotein NBDY. In a reductionist system, NBDY forms liquid droplets in the presence of RNA that can be dissociated by NBDY phosphorylation. While tandem repeat sequences are often understood to undergo phase separation efficiently, the NBDY-RNA system is similar to previously reported peptide-RNA coacervates $^{22}$, and provides support for the notion that intrinsically disordered peptides and small proteins encoded in smORFs may be able to undergo phase separation in vitro or in cells. Furthermore, while we did not directly measure the stoichiometry and specificity of NBDY phosphorylation by PKC in vitro, the insensitivity of NBDY S60A mutantcontaining liquid droplets to PKC suggests that one phosphorylation site on NBDY is likely sufficient to drive liquid phase remixing. This suggests that the overall charge of NBDY is near an inflection point for complex coacervate formation with RNA at the experimental concentrations used, finely tuning NBDY-RNA liquid droplets for responsiveness to phosphorylation. However, NBDY has not been shown to directly interact with RNA in cells, so the physiological relevance of the NBDY-RNA coacervation system remains to be established.

While phase separation in P-bodies is described by a complex phase diagram dependent on a network of RNA-RNA, RNA-protein, and protein-protein interactions ${ }^{40}$, the current study demonstrates that NBDY phosphorylation is an essential driver of P-body dynamics during cell growth and division. While NBDY is phosphorylated at different positions during mitosis and EGFR signaling, both NBDY phosphorylation sites have a similar functional outcome: they are necessary, but not sufficient, for P-body disappearance. Along with the observation that P-body dissociation does not occur in NBDY KO cells, these data are consistent with a model in which NBDY phosphorylation is the switch that drives electrostatic repulsion in the context of phosphorylation of additional P-body proteins during mitosis and EGFR activation, promoting liquid phase re-mixing of the components of macroscopic P-bodies. This model is not at odds with previous reports demonstrating that $N B D Y \mathrm{KO}$ increases the basal number of P-bodies in cells, because $N B D Y$ deletion primarily alters the RNA content of cells, promoting phase separation via increased RNA-RNA and RNA-protein interactions in P-bodies ${ }^{12}$. We propose, therefore, that the cellular role of NBDY is to participate in P-body phase separation, and to dynamically regulate P-body dissociation when phosphorylated. NBDY is therefore a master regulator of P-body dissociation that integrates inputs from multiple signaling pathways that promote cell growth and division. The fate of P-body-resident transcripts after P-body dissociation is of interest in the future; it has been previously proposed that disassembly of membraneless compartments during mitosis is required to ensure equal distribution of proteins and RNAs between two daughter cells ${ }^{4241}$, and we speculate that mitogenic growth factor signaling dissociates P-bodies for a similar reason. In conclusion, these results demonstrate that a 7-kDa microprotein can integrate inputs from different signaling pathways to control the dynamics of a membraneless organelle, and motivate the need to further understand the modifications and interactions of thousands of yet-uncharacterized protein products of smORFs in human cells.

\section{Experimental Section \\ Data analysis}

Two-tailed and equal-variance Student's $t$ test, Mann Whitney $U$ test, Kruskal-Wallis test and analysis of variance (ANOVA) with Dunnett's test were performed using Excel, GraphPad Prism 7 or R. Statistical significance was defined as $\mathrm{P}$ value $<0.05$. Equal variance between samples was established using an $F$-test. P-body counting was performed using ImageJ as previously described ${ }^{44}$.

\section{Recombinant expression, purification and fluorophore labeling of human NBDY}

His-tagged human NBDY and NBDY S61A in pET28a were expressed in E. coli BL21(DE3) cells and purified via Histag affinity chromatography exactly as previously described ${ }^{12}$. For fluorescence microscopy of liquid-liquid phase separated droplets, purified NBDY was labeled with tetramethylrhodamine-5-maleimide (Sigma). NBDY protein was prepared in phosphate buffered saline (PBS) buffer $(\mathrm{pH} 7.4)$ at a concentration of $1 \mathrm{mg} / \mathrm{ml} .1 \mu \mathrm{g}$ of tetramethylrhodamine (TMR)-5-maleimide (Thermo Scientific, Cat. T6027) was dissolved in DMSO, and added to the NBDY solution. The labeling reaction was allowed to proceed for up to two hours at room temperature in the dark. The reaction was stopped by addition of dithiothreitol (DTT) to a final concentration of $4 \mathrm{mM}$. Unconjugated dye was removed using a Sephadex G-25 column in PBS buffer. Labeled NBDY was mixed with non-labeled NBDY at $10 \%$ concentration $(0.1 \mathrm{mg} / \mathrm{ml} \mathrm{TMR} \mathrm{labeled}$ NBDY+0.9 mg/ml NBDY) to give TMR-NBDY.

\section{Turbidity measurements}

Samples were prepared by mixing all components from stock solutions. Polyuridylic acid potassium salt (poly(U), MW $=600-1000 \mathrm{kDa}$, Cat. P9528) and FITC-labeled 40-mer uridine oligonucleotides (FITC-U $\mathrm{U}_{40}$ ) were purchased from Sigma- 
Aldrich and reconstituted in DEPC-treated water. Total cellular RNA was purified from HEK $293 \mathrm{~T}$ cells grown in $15 \mathrm{~cm}$ dishes using Trizol (Invitrogen) following the manufacturer's instructions, followed by DNase I (NEB) treatment, and dissolved in DEPC water. For liquid droplet formation, TMR-NBDY or NBDY was mixed with RNA (poly(U), FITC-U 40, or total cellular RNA) at the indicated concentrations in LLPS buffer (25 mM HEPES, $\mathrm{pH} 7.4,0.5 \mathrm{mM}$ sodium azide, $0.5 \% \mathrm{w} / \mathrm{v}$ Tryptone, $10 \%$ PEG 3350 ) at $30{ }^{\circ} \mathrm{C}$. For the PKC assay, purified TMR-NBDY, NBDY, or NBDY S61A was added to RNA and kinase (PKC $\alpha$ ) in a total volume of $100 \mu \mathrm{L}$ in LLPS buffer with additional components required for PKC $\alpha$ activity $\left(20 \mu \mathrm{M} \mathrm{CaCl}_{2}, 10 \mathrm{mM} \mathrm{DTT}, 100 \mathrm{mM} \mathrm{MgCl}_{2}, 0.5 \mathrm{mg} / \mathrm{ml}\right.$ phosphatidylserine, $\left.100 \mu \mathrm{M} \mathrm{DTT}\right)$ at $30^{\circ} \mathrm{C}$. Turbidity measurements were made in real time using a GENESYS 10S UV-vis spectrophotometer (Thermo Scientific) at a wavelength of $500 \mathrm{~nm}$.

\section{Confocal imaging of liquid droplets}

Samples were prepared by mixing all components from stock solutions in LLPS buffer and spotted on no.1.5 glass coverslips (Fisher Scientific). Confocal images were acquired at room temperature for samples without kinase using a Leica TCS SP8 with PL APO 63x/1.40 oil objective with CORR CS. When kinase was added, images were acquired on an INUBG2AF-GSI2 (Tokai Hit) temperature-controlled stage held at $30^{\circ} \mathrm{C}$.

\section{NMR experiments}

His-tagged human NDBY in pET28a was expressed in E. coli. BL21(DE3) cells grown in M9 minimal medium supplemented with ${ }^{15} \mathrm{NH}_{4} \mathrm{Cl}(1.0 \mathrm{~g} / \mathrm{L})$ purchased from Cambridge Isotope Laboratories (Cat. NLM-467-1). NBDY was purified on Talon metal affinity resin (Takara Bio) followed by cleavage with PreScission protease (Sigma-Aldrich, Cat. GE27-0843-01). NBDY was subsequently further purified by size exclusion chromatography using Superdex 75 (GE Healthcare) and eluted into NMR buffer (50 mM HEPES, pH 7.4, $150 \mathrm{mM} \mathrm{NaCl}, 7 \% \mathrm{D}_{2} \mathrm{O}$ and $1 \mathrm{mM}$ TCEP). For NMR experiments, NBDY was concentrated to $1 \mathrm{mM}$ using Amicon Ultra-15 concentrators (4 kDa molecular weight cut off).

NMR experiments were performed on Varian $600 \mathrm{MHz}$ spectrometers at $293 \mathrm{~K}$. Two-dimensional transverse relaxation optimized spectroscopy ${ }^{1} \mathrm{H}^{15} \mathrm{~N}$ single quantum coherence spectra ${ }^{45}$ were collected with 4096 data points in the direct dimension, $128 \mathrm{t}_{1}$ increments and spectral widths of $12000 \mathrm{~Hz}$ and $2500 \mathrm{~Hz}$ in the direct and indirect dimensions, respectively. Additionally, the ${ }^{1} \mathrm{H}$ transmitter and ${ }^{15} \mathrm{~N}$ offsets are set to water resonance and $120 \mathrm{ppm}$ respectively. Data were processed with NMRPipe and analyzed in nmrFAM-SPARKY ${ }^{46}$.

\section{Cell culture}

HEK 293 T cells were amplified from early-stage stocks prepared from cells purchased from ATCC. HEK 293T cells were cultured in Dulbecco's Modified Eagle Medium (DMEM, Corning, Cat. 10-013-CV) supplemented with 10\% (vol/vol) fetal bovine serum (FBS, Sigma Aldrich, Cat. F4135-500ML) and $100 \mathrm{U} / \mathrm{mL}$ penicillin-streptomycin (Sigma Aldrich, Cat. P4333-100ML). HEK 293T cells bearing NBDY genomic deletion (NBDY KO) were previously described ${ }^{12}$. A549, HCC827, H1975 and H3255 cells were a gift from Craig Crews (Yale University) and were cultured in RPMI-1640 (Corning, Cat. 10-040-CV) medium containing 10\% FBS and $100 \mathrm{U} / \mathrm{mL}$ penicillin-streptomycin. The ATCC Universal mycoplasma detection kit was used to confirm mycoplasma-free status of all cell lines.

\section{Antibodies, Peptides and Proteins}

Primary antibodies used for Western blotting and/or immunofluorescence were: mouse monoclonal anti-FLAG (Sigma, F3165); rabbit polyclonal anti-EDC4 (Sigma, SAB4200114); rabbit polyclonal anti-DCP2 (Novus Biologicals, NBP141070); mouse monoclonal anti-beta actin (Invitrogen, BA3R); rabbit polyclonal anti-DCP1A (Sigma, D5444); rabbit monoclonal anti-DCP1A Alexa Fluor 647 (Abcam, ab209946); rabbit monoclonal anti-EGFR (Abcam, ab40815; rabbit monoclonal anti-EGFR (phospho Y1068) (Abcam, ab40815); mouse monoclonal anti-AKT (Cell Signaling technology, 2966S); rabbit monoclonal anti-AKT (phospho Ser473) (Cell Signaling technology, 4060T); rabbit monoclonal antiERK1/2 (Cell Signaling technology, 4695T); rabbit monoclonal anti-ERK1/2 (Cell Signaling technology, 4695T); rabbit monoclonal anti-ERK1 (phospho T202)+ERK2 (phospho T185) (Abcam, ab201015); rabbit polyclonal anti-NBDY (Covance, YU-342); rabbit polyclonal anti-NBDY(phospho T40) (Covance, YU-352); rabbit polyclonal antiNBDY(phospho S61) (Covance, YU-344). Secondary antibodies for Western blotting were: goat anti-rabbit peroxidase conjugate (Rockland, 611-103-122; Merck, AP132P) and goat anti-mouse peroxidase conjugate (Rockland, 610-1319). Secondary antibody for immunofluorescence was goat anti-rabbit Alexa Fluor 568 (Life Technologies, A-11011).

Synthetic peptides used to generate antibodies against NBDY, NBDY(phospho T40) and NBDY(phospho S61) were as follows: NBDY (amino acids 8-28 of NBDY), phosphorylated Thr40 (amino acids 35-45 of NBDY), and phosphorylated Ser61 (amino acids 54-64 of NBDY). All peptides were synthesized and used as an immunogen in rabbits. All peptides and antibodies were generated by Covance Inc. (Princeton, New Jersey, USA).

\section{Lentivirus production and infection}

Lentivirus was produced essentially as previously reported ${ }^{47}$. HEK $293 \mathrm{~T}$ cells were grown in 10 -cm culture dishes to $60 \%$ confluency, then co-transfected using Lipofectamine 2000 (Thermo Scientific, 11668019) with pLJM1 encoding the transgene to be stably expressed, along with accessory plasmids pMD2.G and psPAX2, and growth media was replaced 
after $5 \mathrm{~h}$. Forty-eight hours later, lentivirus-containing media was harvested, and cell debris was removed by passing through a $0.45-\mu \mathrm{m}$ filter prior to aliquoting and flash freezing.

Stable cell lines were generated by treating HEK293T or HEK293T NBDY KO cells, grown in 6-well plates to 75\% confluency, with $\sim 20 \mu \mathrm{L}$ of the appropriate lentivirus-containing media. A polyclonal population of stable cells were selected with $2 \mathrm{ng} / \mathrm{ml}$ puromycin (Sigma, Cat. P9620), and, forty-eight hours later, cells were harvested and transgene expression was confirmed by Western blotting.

\section{NBDY immunoprecipitation and phosphoproteomics}

To detect NBDY phosphorylation in cells undergoing mitosis, 70\% confluent HEK 293T cells in a 10-cm dish released from $2 \mathrm{mM}$ thymidine block for twenty-four hours were transfected with FLAG-tagged NBDY in pcDNA3 with OptiMEM medium. Transfections were carried out using Lipofectamine 2000 (Thermo Fisher, 11668019) and $10 \mu \mathrm{g}$ DNA per $10 \mathrm{~cm}$ dish of cells. After 6 hours, Opti-MEM medium was removed, and nocodazole (Sigma, Cat. M1404) was added to a final concentration of $100 \mathrm{ng} / \mathrm{ml}$. To assess NBDY phosphorylation during epidermal growth factor stimulation, $70 \%$ confluent HEK 293T cells in a $10 \mathrm{~cm}$ dish were transfected with FLAG-tagged NBDY in pcDNA3, and 24 hours later were treated with $20 \mathrm{ng} / \mathrm{ml}$ EGF (Sigma, Cat. E9644) for $30 \mathrm{~min}$. NBDY was subsequently immunopurified from both experimental sets of cells prior to phosphoproteomic analysis following a previously published protocol. Briefly, cells were harvested and lysed on ice using $400 \mu \mathrm{L}$ Tris-buffered saline (TBS) with 1\% Triton X-100 supplemented with Roche Complete protease inhibitor cocktail tablets. After $3 \times$ FLAG peptide (Sigma, Cat. F4799) elution of FLAG-NBDY from anti-FLAG agarose beads (clone M2, Sigma, Cat. A2220) in TBS, the entire eluate from each sample was loaded on an SDS-PAGE gel, separated, visualized with Coomassie stain and imaged.

Protein-containing gel slices were digested with trypsin as previously described ${ }^{13}$. The resulting peptide mixtures were extracted from the gel and dried, subjected to ethyl acetate extraction to remove residual detergent, and re-suspended in $15 \mu \mathrm{l}$ of 3:8 70\% formic acid: $0.1 \%$ TFA. A $10 \mu \mathrm{L}$ aliquot of each sample was injected onto a pre-packed column attached to a nanoAcquity UPLC (Waters) in-line with an LTQ Orbitrap Velos (Thermo Scientific). Samples were trapped for 15 min with a flow rate of $2 \mu \mathrm{l} / \mathrm{min}$ on a 100 -micron ID trap column packed for $5 \mathrm{~cm}$ with $5 \mu \mathrm{m}$ Magic C18 AQ beads (Waters), and peptides were chromatographed on a $20 \mathrm{~cm}$ 75-micron ID analytical column (New Objective) packed inhouse with $3 \mu \mathrm{m}$ Magic C18 AQ beads (Waters). A NanoAcquity pump (Waters) eluted peptides at $300 \mathrm{nl} / \mathrm{min}$ flow rate as follows: Buffer A: water/0.1\% formic acid; Buffer B: MeCN/0.1\% formic acid; Gradient: 0\% to $95 \%$ buffer B over 90 minutes. Tandem mass spectrometry (MS) was performed as follows: The full MS was collected over the range of 298$1750 \mathrm{~m} / \mathrm{z}$ with a resolution of 30,000 . MS/MS data were collected using a top 10 high-collisional energy dissociation method in data-dependent mode with a normalized collision energy of $33.0 \mathrm{eV}$ and a $2.0 \mathrm{~m} / \mathrm{z}$ isolation window. The first mass was $100 \mathrm{~m} / \mathrm{z}$ in fixed mode. MS/MS resolution was 7500 and dynamic exclusion was $60 \mathrm{~s}$.

Mass spectra were analyzed using Mascot (version 2.5.0.1). We searched a database comprising human Uniprot proteins (version 2019) with the NBDY protein sequence appended. Phosphorylation of Ser, Thr and Tyr, oxidation of methionine and N-terminal acetylation were set as variable modifications. The MS1 mass error was set to $20 \mathrm{ppm}$, and MS/MS peak error was set at a maximum of 0.6 Da. Up to two missed cleavages were permitted. The maximum false discovery rate (FDR) was set to 0.01 for peptide and protein identifications. The minimum peptide length was set at five amino acids.

\section{Western blotting}

For validation of NBDY phosphorylation sites detected by mass spectrometry, FLAG-NBDY immunoprecipitants were prepared as above and resolved on 15\% SDS-PAGE gel containing $25 \mu \mathrm{M}$ of Phos-tag acrylamide (Wako, Cat. AAL-107) and $125 \mu \mathrm{M} \mathrm{MnCl}_{2}$. After electrophoresis, Phos-tag acrylamide gels were washed with transfer buffer containing $10 \mathrm{mM}$ EDTA for $30 \mathrm{~min}$ with gentle shaking and then with transfer buffer without EDTA for 30 min according to the manufacturer's protocol. Immunoblots were blocked with 3\% BSA in TBS-T for 1 hour at room temperature (RT), then probed with both anti-NBDY (Covance, YU-342), anti-NBDY(phospho T40) (Covance, YU-352) and antiNBDY(phosphorS61) (Covance, YU-344) overnight at $4{ }^{\circ} \mathrm{C}$. Control cell lysates were treated with $4 \mathrm{U} / \mu \mathrm{l}$ of Lambda phosphatase (NEB, P0753S) at $30{ }^{\circ} \mathrm{C}$ for 30 minutes before immunoprecipitation and Phos-tag SDS-PAGE and immunoblotting analysis.

For validation of NBDY phosphorylation downstream of CDKs and EGFR, proteins were transferred to nitrocellulose membranes followed by a standard Western blotting protocol ${ }^{11}$. Briefly, immunoblots were blocked with $3 \%$ BSA in TBS$\mathrm{T}$ for 1 hour at RT and probed with primary antibodies against pathway (phospho)proteins in 3\% BSA in TBS-T overnight at $4{ }^{\circ} \mathrm{C}$. The membrane was washed three times with TBS-T, probed with secondary antibodies in $3 \%$ BSA in TBS-T for 1 hour at RT, and washed three times with TBS-T before development with Clarity ECL Western Blotting Substrate (BioRad, Cat. 1705060) and imaging.

\section{Kinase inhibition assay}

To identify the kinase that phosphorylates NBDY during mitosis, HEK 293T cells released from 2 mM thymidine block for twenty-four hours were transfected with FLAG-tagged NBDY in pcDNA3 with Opti-MEM medium. Six hours later, Opti-MEM was removed, and a CDK inhibitor (1 $\mu$ M AT7519 (Selleck Chemicals, Cat. S1524)) or PI3K inhibitor (1 $\mu$ M 
wortamanin (Selleck Chemicals, Cat. S2758)) was added with $100 \mathrm{ng} / \mathrm{ml}$ nocodazole in complete media. Cells were harvested and suspended in $1 \mathrm{~mL}$ lysis buffer (Tris-buffered saline (TBS) with 1\% Triton X-100 and Roche Complete protease inhibitor cocktail tablets). Then cells were sonicated (50\% intensity, $5 \mathrm{~s}$ pulse with $25 \mathrm{~s}$ rest, $5 \times$, MICROSON XL 2000) on ice followed by centrifugation at $21,130 \times \mathrm{g}, 4^{\circ} \mathrm{C}, 10 \mathrm{~min}$. The proteins were transferred to nitrocellulose membranes followed by a standard Western blotting protocol ${ }^{11}$ as described above.

To identify the kinase that phosphorylates NBDY during EGF stimulation, HEK 293T cells transfected with FLAGtagged NBDY were treated with kinase inhibitors targeting EGFR (10 $\mu \mathrm{M}$ AG1478 (Selleck Chemicals, Cat. S2728)), MEK (5 $\mu$ M U0126 (Selleck Chemicals, Cat. S1102)), PI3K (1 $\mu$ M wortamannin (Selleck Chemicals, Cat. S2758)), or CDK (1 $\mu$ M AT7519 (Selleck Chemicals, Cat. S1524)) for 4 hours; an inhibitor targeting PKC $(10 \mu \mathrm{M}$

Bisindolylmaleimide II (Cayman Chemicals, Cat. 11020)) was applied to cells for 8 hours. Following kinase inhibitor or vehicle treatment, cells were stimulated by adding $20 \mathrm{ng} / \mathrm{ml}$ EGF for $30 \mathrm{~min}$. Cells were harvested and lysed, and proteins were subjected to a standard Western blotting protocol ${ }^{11}$ as described above.

\section{In vitro kinase assay}

In vitro assays of NBDY phosphorylation at S61 by purified kinases were performed essentially as described previously $^{48}$. Briefly, $1 \mu \mathrm{g}$ purified NBDY was incubated with PKC $\alpha(200 \mathrm{ng}$; Thermo Fisher, Cat. A41891) in a total assay volume of $20 \mu \mathrm{l}$ of buffer containing $20 \mathrm{mM}$ HEPES, pH 7.4, $20 \mu \mathrm{M} \mathrm{CaCl}_{2}, 10 \mathrm{mM} \mathrm{DTT}, 100 \mathrm{mM} \mathrm{MgCl}_{2}, 100 \mu \mathrm{M}$ ATP, $0.6 \mathrm{mg} / \mathrm{ml}$ phosphatidylserine and $0.06 \mathrm{mg} / \mathrm{mL}$ diacylglycerol. Grk2 (200 ng; Thermo Fisher, Cat. PV3361) and GSK3 $\beta$ (200 ng; Thermo Fisher, Cat. PV3365) were incubated with $1 \mu \mathrm{g}$ purified NBDY in a total assay volume of $20 \mu \mathrm{L}$

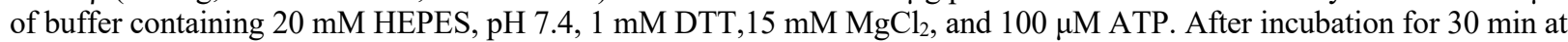
$30{ }^{\circ} \mathrm{C}$, reactions were stopped by addition of SDS loading buffer and proteins were separated by SDS-PAGE.

Phosphorylation of NBDY was determined by Western blotting with rabbit polyclonal anti-NBDY(phospho S61) (Covance, YU-344), using rabbit polyclonal anti-NBDY (Covance, YU-342) antibody as a loading control.

\section{Immunoprecipitation of NBDY from lung cancer cell lines}

For agarose bead antibody conjugation, $100 \mu \mathrm{L}$ NHS-activated agarose slurry (Thermo Scientific, Cat. 26200) was washed twice with PBS buffer (pH 7.4). Subsequently $30 \mu \mathrm{L}$ rabbit polyclonal anti-NBDY (Covance, YU-342) (1 mg/ml) in PBS buffer was added, and the resulting mixture was incubated on ice for one hour. Unreacted NHS-beads were quenched with $1 \mathrm{M}$ ethanolamine $(\mathrm{pH} 7.4)$ for another 15 minutes. The resulting resin was washed with PBS (5x) and stored in PBS on ice at $4^{\circ} \mathrm{C}$ for 12 hours. A549, HCC827, H1975 and H3255 were grown to 60-70\% confluence in 15 -cm dishes (treated with $5 \mu \mathrm{M}$ erlotinib (Sigma, Cat. SML2156) or vehicle for 24 hours) were harvested and lysed using $400 \mu 1$ lysis buffer (TBS with 1\% Triton X-100 and Roche Complete protease inhibitor cocktail tablets). Cells were lysed on ice for $20 \mathrm{~min}$ followed by centrifugation at 14,000 r.p.m., $4^{\circ} \mathrm{C}, 15 \mathrm{~min}$. A $50 \mu$ laliquot of anti-NBDY agarose beads was suspended in the cell lysate supernatant. Bead suspensions were rotated at $4^{\circ} \mathrm{C}$ for 1 hour and washed 3 times with TBS-T. Elution was carried out by boiling the beads in $30 \mu \mathrm{L}$ of $3 \times$ protein loading buffer $(0.15 \mathrm{M}$ Tris- $\mathrm{HCl}, \mathrm{pH} 8.0,15 \%$ glycerol, $50 \mathrm{mg} / \mathrm{mL}$ SDS, $50 \mathrm{mg} / \mathrm{mL}$ DTT, $50 \mu \mathrm{g} / \mathrm{mL}$ bromophenol blue). The protein was boiled for 10 minutes, diluted to $1 \times$. Beads were removed by centrifugation, and the entire supernatant was loaded on a 15\% SDS-PAGE gel. Western blotting with rabbit polyclonal anti-NBDY(phospho S61) (Covance, YU-344), using rabbit polyclonal anti-NBDY (Covance, YU-342) antibody as a loading control.

\section{P-Body imaging}

HEK 293 T cells were grown on fibronectin-coated glass coverslips (AmScope, CS-R18-100, 18 mm diameter round microscope glass cover slides) in a 12 -well plate to $70 \%$ confluency. Cells were fixed with $10 \%$ neutral buffered formalin (Fisher Scientific), permeabilized with methanol at $-20{ }^{\circ} \mathrm{C}$, and blocked with blocking buffer ( $3 \%$ BSA in PBS) for $1 \mathrm{~h}$ at room temperature. Cells were stained with rabbit polyclonal anti-NBDY (Covance, YU-342) at a 1:100 dilution (volume: volume) in blocking buffer overnight at $4{ }^{\circ} \mathrm{C}$, followed by 3 consecutive washes with PBS. Goat anti-rabbit Alexa Fluor 568 (Life Technologies, A-11011) was subsequently applied at a 1:1000 dilution in blocking buffer for 1 to 4 hours at room temperature in the dark, followed by 5 times with PBS washes. Anti-DCP1A Alexa Fluor 647(Abcam, ab209946) was then added at 1:1000 dilution and incubated for 1 hour, followed by 3x PBS washes. For quantifying P-bodies, cells were stained with rabbit anti-DCP1A (Sigma-Aldrich, D5444) at a 1:1000 dilution in blocking buffer overnight at $4{ }^{\circ} \mathrm{C}$, followed by $3 x$ PBS washes and incubation with goat anti-rabbit Alexa Fluor 568. Cells were post-fixed with 10\% buffered formalin, nuclei were stained with DAPI (EMD Millipore, Cat. 268298, 1:20000 dilution in 1x PBS), and imaging was performed on a laser scanning confocal microscope (Leica TCS SP8) with PL (field planarity) APO (apochromatic) 63x/1.40 oil, CS2 and PL APO 100x/1.44 oil, CORR (correction collar) CS (confocal scanning).

\section{Safety statement}

The third-generation lentivirus system was utilized for establishing the stable cell lines for improved safety. All experiments were performed in designated laminar flow biological safety hood with posted biohazard label. All consumables in contact with virus or virus-containing cells were decontaminated with $10 \%$ bleach and discarded in BL2 
waste. Generation of aerosols was stringently avoided. No other unusually high or unexpected chemical or biological safety hazards were encountered.

\section{ASSOCIATED CONTENT \\ SUPPORTING INFORMATION}

The Supporting Information is available free of charge at https://pubs.acs.org

Supporting Figures 1-5

Movie S1, NBDY-RNA liquid droplet fluorescence microscopy

Movie S2, NBDY-RNA coacervate phosphorylation and remixing

Table S1, NBDY phosphoproteomics data

\section{Accession Codes}

Phosphoproteomic data are available from the PRIDE repository under accession number PXD026098.

\section{Author Contributions}

The manuscript was written through contributions of all authors. All authors have given approval to the final version of the manuscript.

\section{Acknowledgments}

We thank all members of the Slavoff lab, Ken Loh, Mark Lemmon, Ben Turk and Jesse Rinehart for helpful discussions. This work was supported by the Searle Scholars Program, an Odyssey Award from the Richard and Susan Smith Family Foundation, NIH R01GM122984, and Yale University West Campus start-up funds (to S.A.S.). A. K. (5T32GM06754 3-12) was in part supported by an NIH Predoctoral Training Grant. 
a

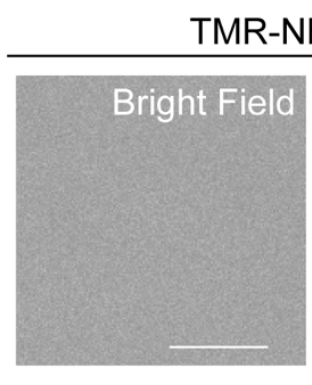

b

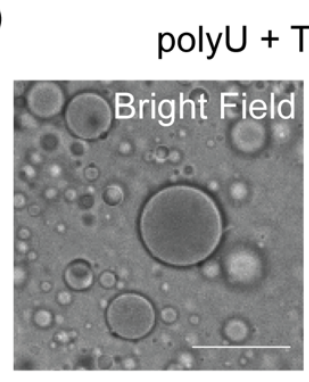

d
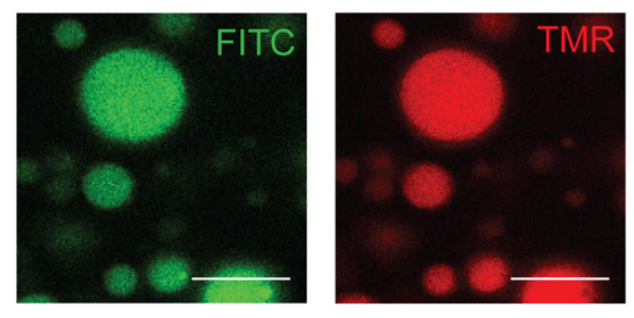

e
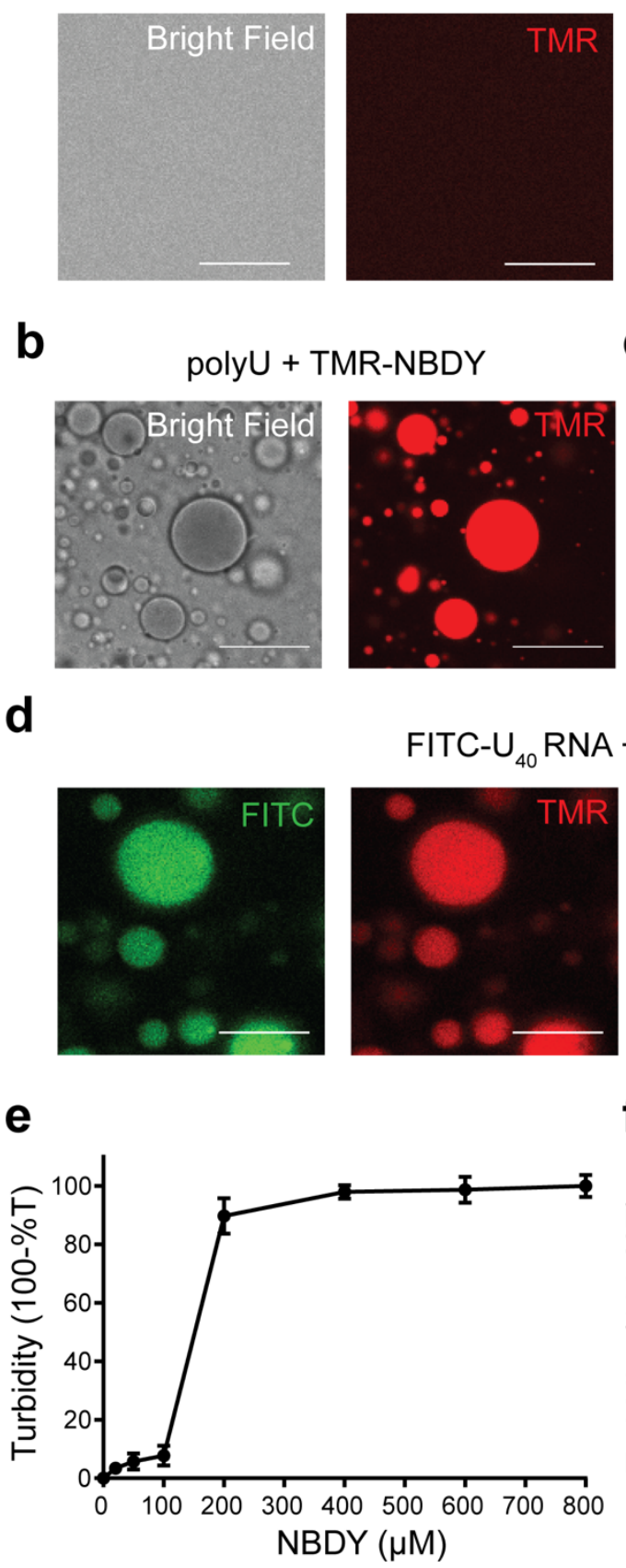

RNA only

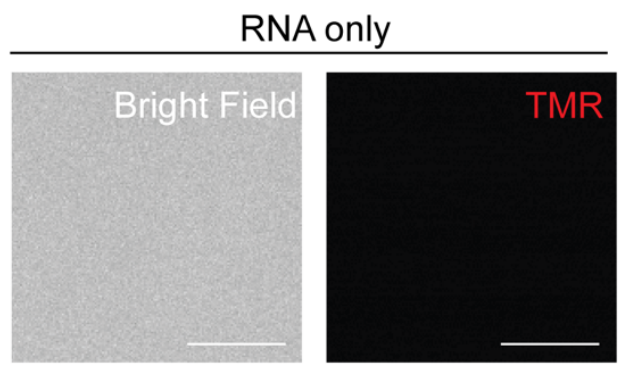

C Cellular RNA + TMR-NBDY
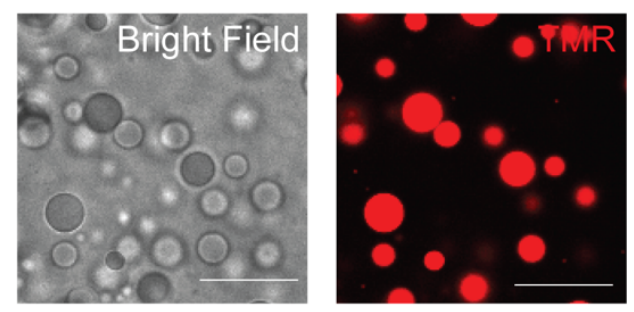

FITC-U ${ }_{40}$ RNA + TMR-NBDY
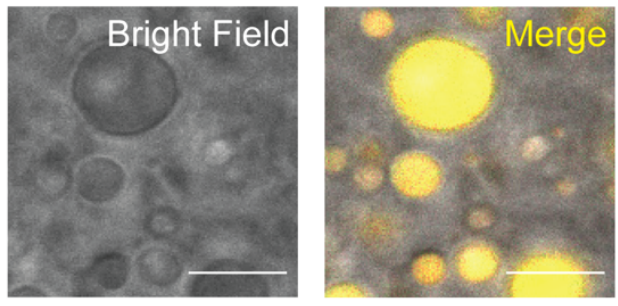

$\mathbf{f}$

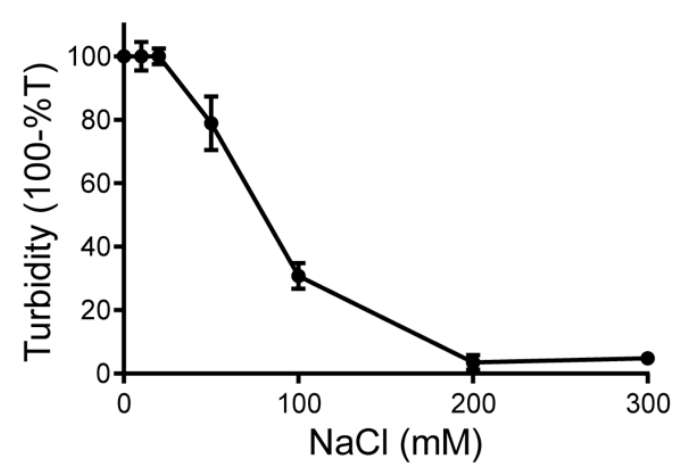

Figure 1| Characterization of RNA/NBDY coacervates. (a) TMR-NBDY or polyU RNA alone do not form phase separated liquid droplets. Transmission brightfield (left) and fluorescence images (right). (b,c) Transmission brightfield (left) and fluorescence images (right) show coacervate phase droplets formed by $0.4 \% \mathrm{w} / \mathrm{v}$ polyU RNA and $200 \mu \mathrm{M}$ TMR-NBDY (b) or by $0.5 \mu \mathrm{g} / \mu \mathrm{L}$ HEK $293 \mathrm{~T}$ RNA and $200 \mu \mathrm{M}$ TMR-NBDY (c). (d) $0.5 \mu \mathrm{g} / \mu \mathrm{L}$ FITC-labelled $\mathrm{U}_{40}$ RNA and $200 \mu \mathrm{M}$ TMR-NBDY colocalize in coacervate droplets. For all panels, the buffer used was $25 \mathrm{mM} \mathrm{HEPES,} \mathrm{pH} \mathrm{7.4,} 0.5 \mathrm{mM}$ sodium azide, $0.5 \% \mathrm{w} / \mathrm{v}$ Tryptone, $10 \%$ PEG3350 at $30^{\circ} \mathrm{C}$. (e,f) Turbidity plot of polyU/NBDY coacervates at increasing concentration of NBDY (e) or $\mathrm{NaCl}$ (f). The concentration of polyU used was $0.4 \% \mathrm{w} / \mathrm{v}$ polyU in all cases, and $200 \mu \mathrm{M}$ NBDY was used in (f). Error bars in turbidity plots represent the standard deviation of three independent trials. For all panels, the buffer used was $25 \mathrm{mM} H E P E S$, pH 7.4, $0.5 \mathrm{mM}$ sodium azide, $0.5 \% \mathrm{w} / \mathrm{v}$ Tryptone, $10 \%$ PEG3350 at $30{ }^{\circ} \mathrm{C}$. 


\section{a}

NBDY microprotein: MGDQPCASGRSTLPPGNAREAKPPKKRCLLAPRWDYPEGTPNGGSTTLPSAPPPASAGLKSHPPPPEK

b
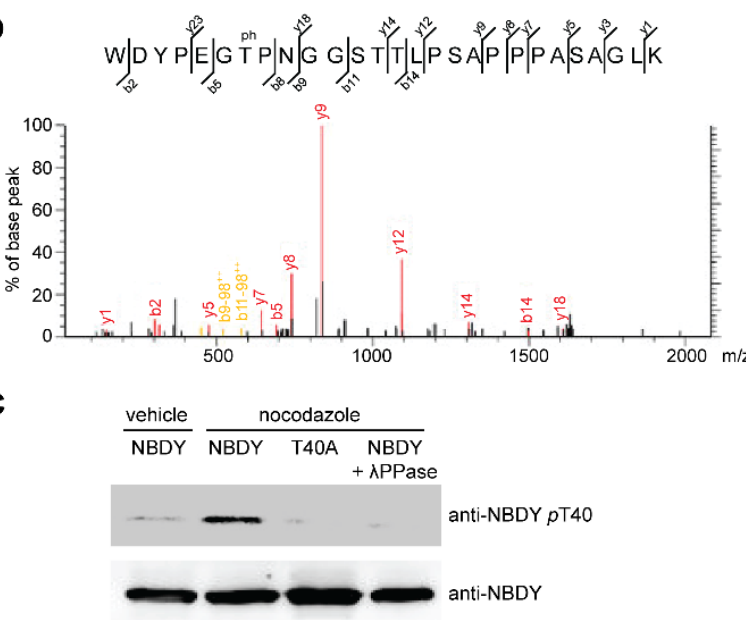

$\mathbf{e}$

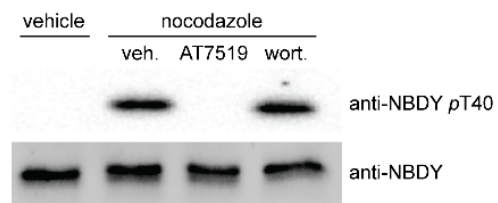

d

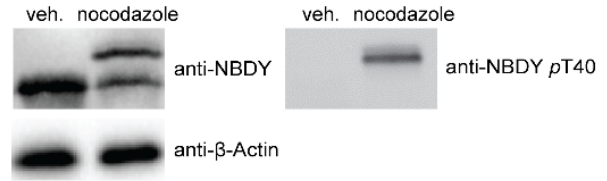

f

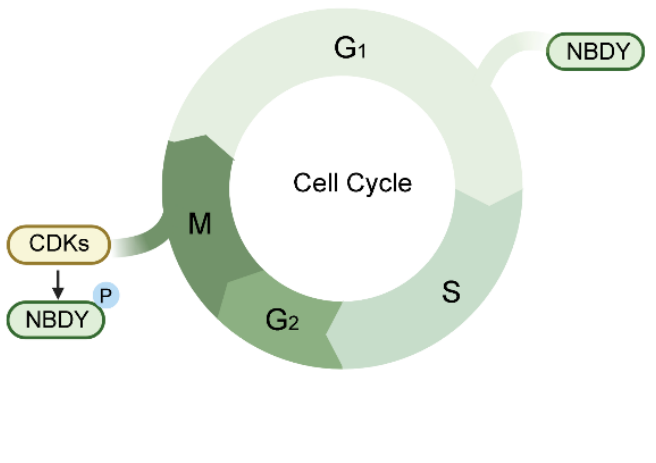

Figure 2| NBDY is phosphorylated at T40 downstream of cyclin-dependent kinases (CDKs) at the G2/M checkpoint. (a) Mitosis-specific NBDY phosphorylation site (red). (b) NBDY phosphorylation at T40 (pT40) after nocodazole treatment identified by LC-MS/MS. Red peaks, y-ions; yellow peaks, b-ions. c) NBDY pT40 antibody validation. Immunopurified samples were prepared from HEK 293T cells transiently expressing WT or T40A FLAG-NBDY with or without nocodazole treatment. $\lambda$ PPase indicates treatment with $\lambda$-phosphatase before SDS-PAGE. (d) Phos-tag PAGE and Western blotting validation of NBDY pT40. Immunopurified samples were prepared from HEK 293T cells transiently expressing FLAG-NBDY with or without nocodazole treatment. $\beta$-Actin, loading control. (e) Phosphorylation of NBDY is regulated by CDKs. Cells expressing FLAG-NBDY were treated with nocodazole in the presence of a CDK inhibitor (AT7519) or a PI3K inhibitor (wortmannin) as a negative control before IP and western blotting. (f) Timing of NBDY phosphorylation at T40 downstream of CDKs at the G2/M checkpoint of the cell cycle. 
a

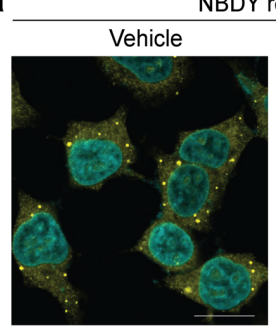

NBDY rescue

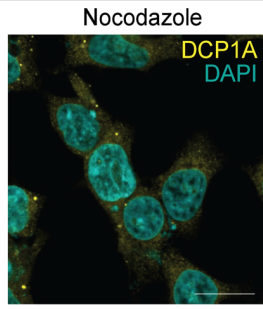

NBDY T40A rescue

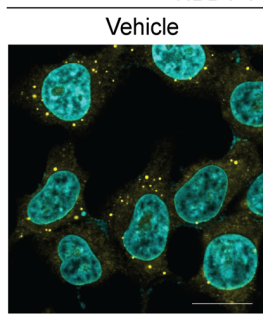

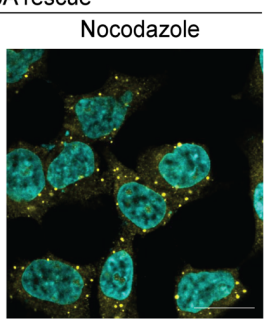

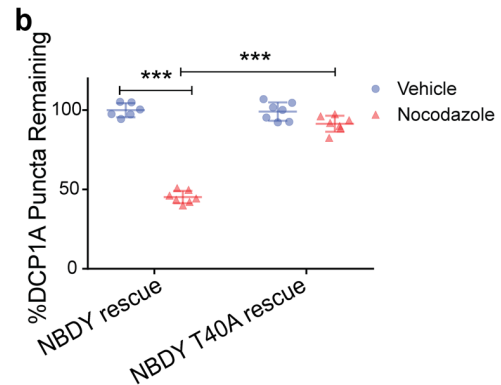

Figure 3| NBDY phosphorylation at T40 is required for P-body disappearance at the G2/M checkpoint. P-body disappearance during mitosis requires NBDY phosphorylation at T40. P-body numbers in nocodazole or vehicle-treated cells expressing NBDY or NBDY T40A were imaged via immunofluorescence. Yellow, anti-DCP1A immunofluorescence; blue, DAPI. Six fields of view ( $>180$ cells) were used to quantitate average P-bodies per cell in each condition (b). Data represent mean values from each field of view \pm s.e.m, and significance was evaluated with one-way ANOVA. ${ }^{* * *} \mathrm{P}<0.001$, Dunnett's test. Scale bars, $20 \mu \mathrm{m}$. 
b

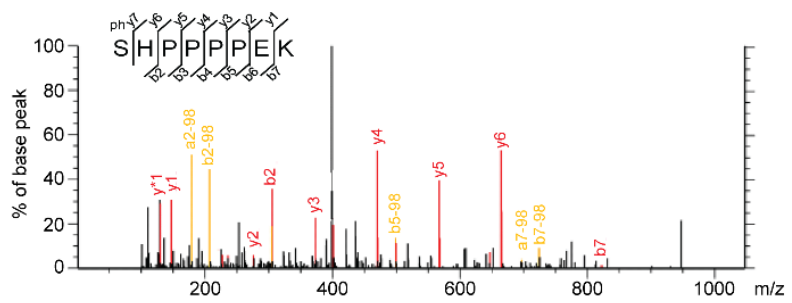

d

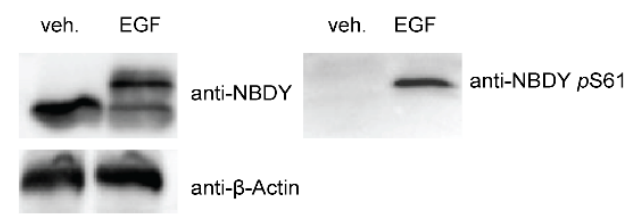

C

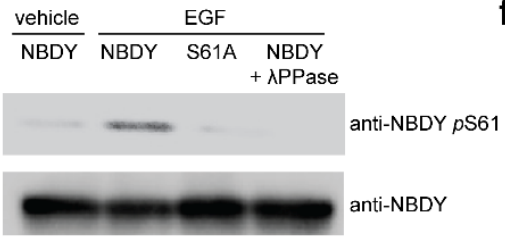

f
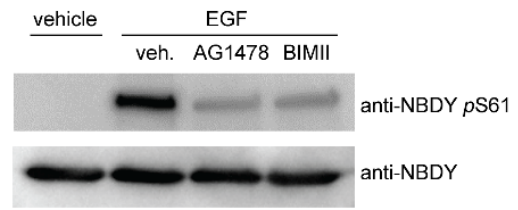

g

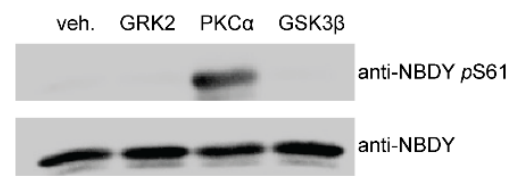

e

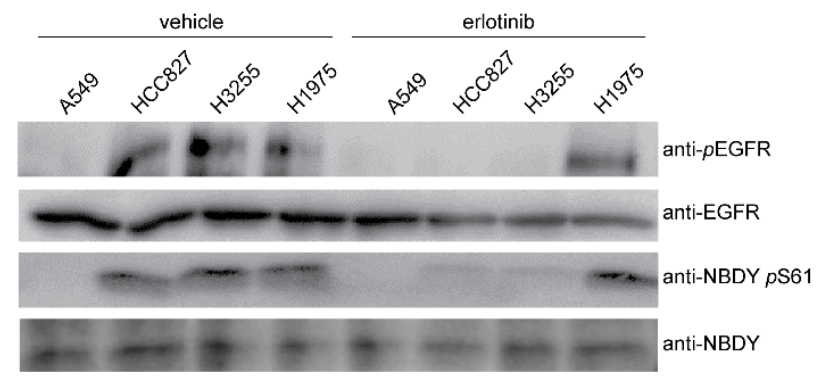

h

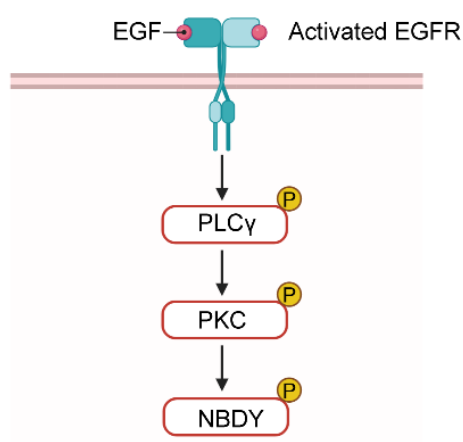

Figure 4| NBDY is phosphorylated at S61 downstream of protein kinase C (PKC) during EGFR signaling. (a) NBDY phosphorylation site observed during EGFR signaling (red). (b) EGF-dependent NBDY phosphorylation site identified by LCMS/MS. Red peaks, y-ions; yellow peaks, b-ions. (c) Validation of anti-NBDY pS61 antibody. Extracts were prepared from HEK 293 T cells transiently expressing FLAG-NBDY or FLAG-NBDY S61A with or without EGF treatment. $\lambda$ PPase indicates treatment with $\lambda$-phosphatase before SDS-PAGE. (d) Phos-tag PAGE and Western blotting validation of NBDY pS61.

Transiently expressed FLAG-NBDY was immunopurified from HEK 293T cells with or without EGF treatment. $\beta$-Actin, loading control. (e) Endogenous NBDY pS61 was assayed in lung cancer cells with wild-type EGFR (A549), activating EGFR mutation (HCC827, H3255), or activating EGFR mutation and erlotinib resistance (H1975) in the presence of vehicle or erlotinib. (f) NBDY pS61 is regulated by PKC. Extracts were prepared from FLAG-NBDY expressing cells treated with vehicle, or with EGF in the presence of vehicle, EGFR inhibitor (AG1478) or PKC inhibitor (BIMII, bisindolylmaleimide II) followed by immunopurification and Western blotting. (g) In vitro kinase assay. Purified NBDY was incubated with purified control kinases (GRK2, GSK3 $\beta$ ) or PKC $\alpha$ in the presence of ATP, then subjected to Western blotting. (h) Model: NBDY is phosphorylated at S61 downstream of PKC during EGFR signaling. 
a

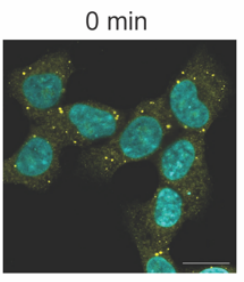

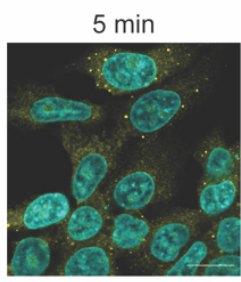
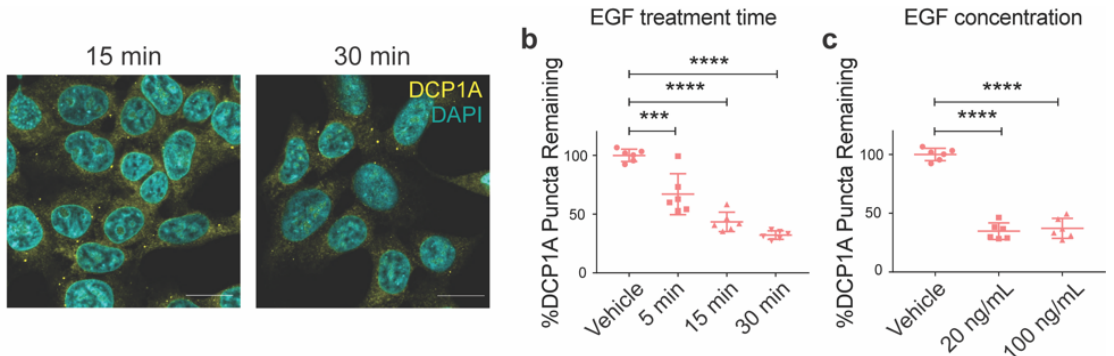

Figure 5| P-body disappearance during EGF stimulation. (a,b) EGF (20 ng/ml) induces P-body disassembly. Six fields of view ( $>180$ cells) were used to quantitate average P-bodies per cell at each timepoint (b). Data represent mean \pm s.e.m, and significance was evaluated with one-way ANOVA. ${ }^{* * *} \mathrm{P}<0.001,{ }^{*} * * * \mathrm{P}<0.0001$, Dunnett's test. (c) EGF concentration dependence for P-body dissociation. Six fields of view ( $>180$ cells) were used to quantitate P-bodies per cell at each EGF concentration. Data represent mean (P-bodies per cell calculated from each field of view) \pm s.e.m, and significance was evaluated with one-way ANOVA. ****P $<0.0001$, Dunnett's test. 
a
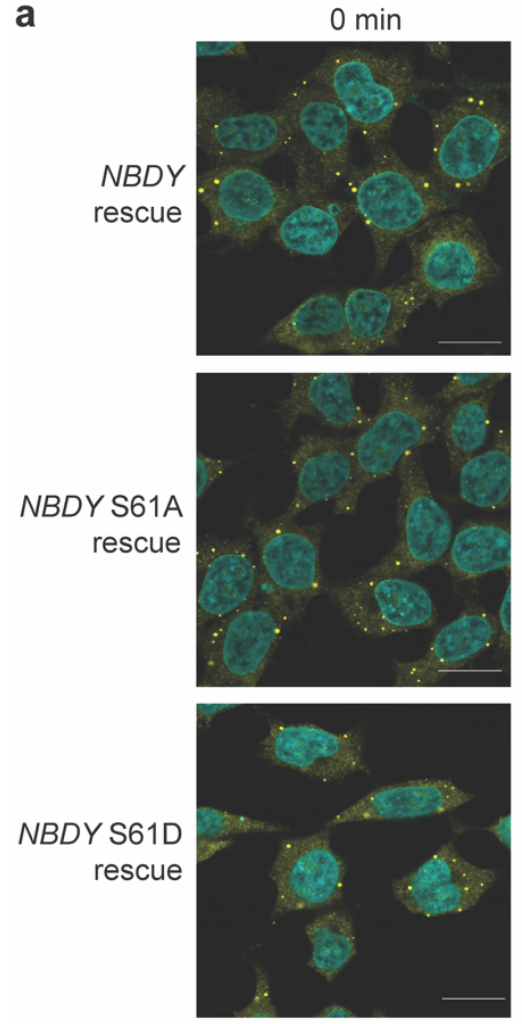
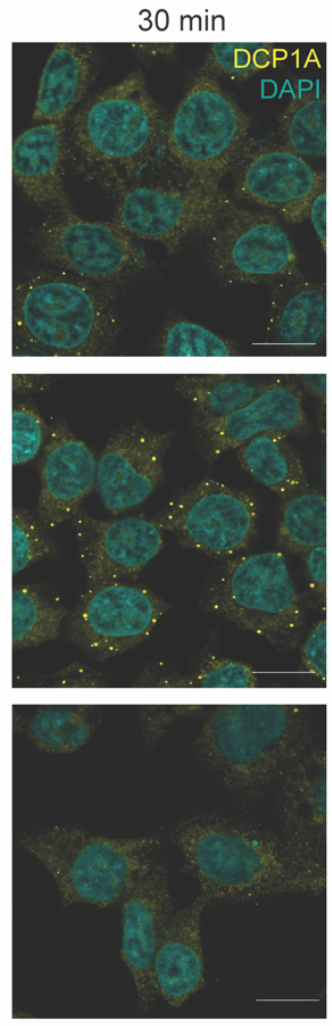

b

P-body numbers in the absence of EGF

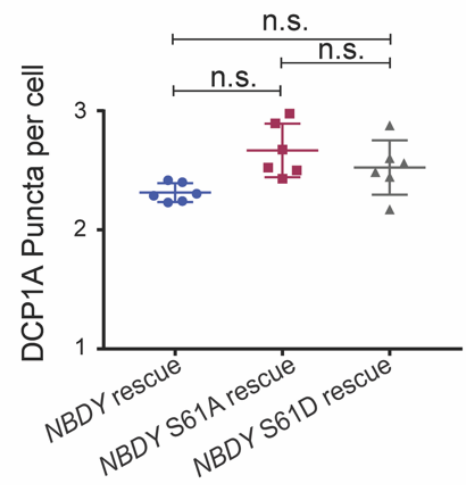

C

P-body numbers in the presence of EGF

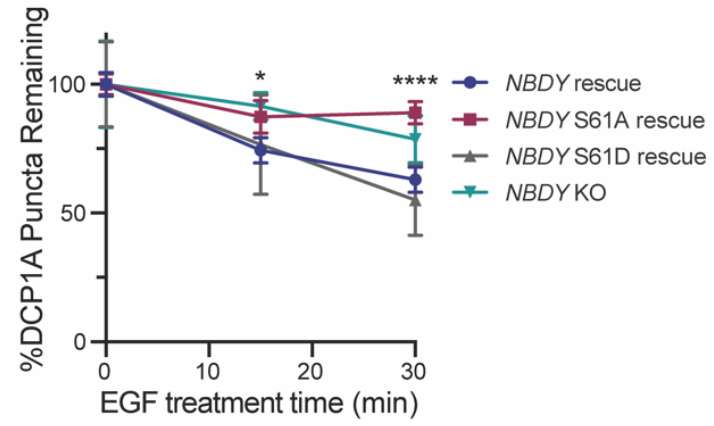

Figure 6| NBDY phosphorylation at S61 is required for P-body disappearance during EGFR signaling. P-bodies were visualized in NBDY rescue, $N B D Y$ KO, NBDY S61A rescue and NBDY S61D rescue cell lines after treatment with EGF or vehicle. Yellow, anti-DCP1A immunofluorescence; blue, DAPI. Six fields of view ( $>180$ cells) were used to quantitate average P-bodies per cell in untreated (b) or EGF-treated cells at indicated time points (c). Data represent mean values \pm s.e.m, and significance was evaluated with one-way ANOVA. n.s., not significant; ${ }^{*} \mathrm{P}<0.05 ; * * * * \mathrm{P}<0.0001$, Dunnett's test. Scale bars, 20 $\mu \mathrm{m}$. 
a
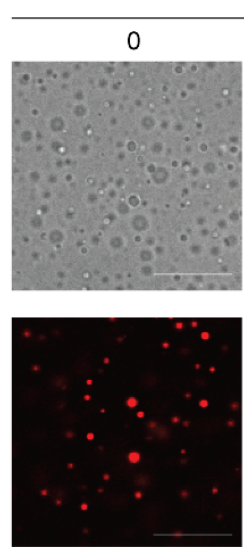

Time (min)
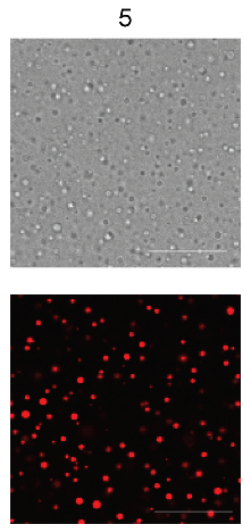

b

15
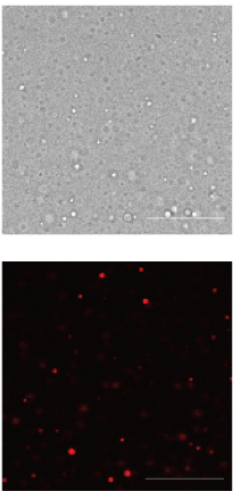

30
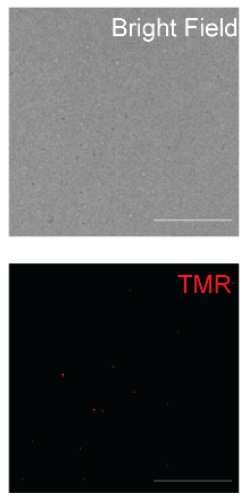

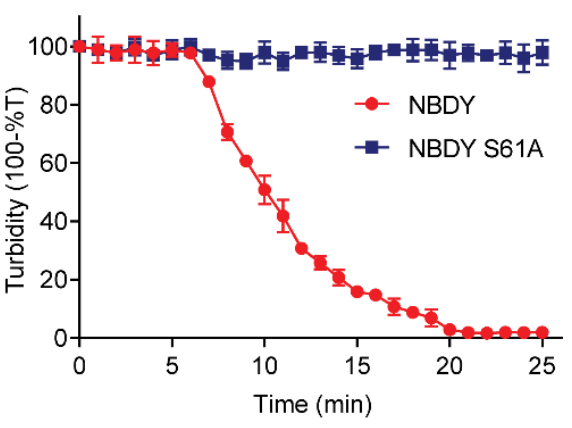

Figure 7| Phosphorylation at $\mathbf{S 6 1}$ by $\mathbf{P K C}$ in vitro dissociates NBDY-RNA liquid droplets. (a) PolyU/NBDY coacervate dissolution by PKC activity. Top row: transmission brightfield images. Bottom row: TMR-NBDY fluorescence at the same time points. Buffer: $25 \mathrm{mM}$ HEPES, pH 7.4, $20 \mu \mathrm{M} \mathrm{CaCl}_{2}, 10 \mathrm{mM}$ DTT, $100 \mathrm{mM} \mathrm{MgCl}_{2}, 0.5 \mathrm{mg} / \mathrm{ml}$ phosphatidylserine, $100 \mu \mathrm{M}$ DTT, $0.5 \mathrm{mM}$ sodium azide, $0.5 \% \mathrm{w} / \mathrm{v}$ Tryptone, $10 \%$ PEG3350 at $30^{\circ} \mathrm{C}$. Scale bars, $10 \mu \mathrm{m}$. (b) Solution turbidity as a function of time for purified wild-type (red) or S61A NBDY (blue) protein in the presence of PKC. Error bars represent the standard deviation of three independent trials. Buffer: $25 \mathrm{mM}$ HEPES, pH 7.4, $20 \mu \mathrm{M} \mathrm{CaCl}_{2}, 10 \mathrm{mM} \mathrm{DTT}, 100 \mathrm{mM} \mathrm{MgCl}, 0.5 \mathrm{mg} / \mathrm{ml}$ phosphatidylserine, $100 \mu \mathrm{M}$ DTT, $0.5 \mathrm{mM}$ sodium azide, $0.5 \% \mathrm{w} / \mathrm{v}$ Tryptone, $10 \% \mathrm{PEG} 3350$ at $30{ }^{\circ} \mathrm{C}$. 


\section{References:}

1. Basrai, M. A.; Hieter, P.; Boeke, J. D., Small open reading frames: beautiful needles in the haystack. Genome Res 1997, 7 (8), 768-71.

2. Martinez, T. F.; Chu, Q.; Donaldson, C.; Tan, D.; Shokhirev, M. N.; Saghatelian, A., Accurate annotation of human proteincoding small open reading frames. Nat Chem Biol 2020, 16 (4), 458-468.

3. Orr, M. W.; Mao, Y.; Storz, G.; Qian, S. B., Alternative ORFs and small ORFs: shedding light on the dark proteome. Nucleic Acids Res 2020, 48 (3), 1029-1042.

4. Brunet, M. A.; Leblanc, S.; Roucou, X., Reconsidering proteomic diversity with functional investigation of small ORFs and alternative ORFs. Exp Cell Res 2020, 393 (1), 112057.

5. Keeling, D. M.; Garza, P.; Nartey, C. M.; Carvunis, A. R., The meanings of 'function' in biology and the problematic case of de novo gene emergence. Elife 2019, 8.

6. $\quad$ Carvunis, A. R.; Rolland, T.; Wapinski, I.; Calderwood, M. A.; Yildirim, M. A.; Simonis, N.; Charloteaux, B.; Hidalgo, C. A.; Barbette, J.; Santhanam, B.; Brar, G. A.; Weissman, J. S.; Regev, A.; Thierry-Mieg, N.; Cusick, M. E.; Vidal, M., Proto-genes and de novo gene birth. Nature 2012, 487 (7407), 370-4.

7. Vakirlis, N.; Acar, O.; Hsu, B.; Castilho Coelho, N.; Van Oss, S. B.; Wacholder, A.; Medetgul-Ernar, K.; Bowman, R. W., 2nd; Hines, C. P.; lannotta, J.; Parikh, S. B.; McLysaght, A.; Camacho, C. J.; O'Donnell, A. F.; Ideker, T.; Carvunis, A. R., De novo emergence of adaptive membrane proteins from thymine-rich genomic sequences. Nat Commun 2020, 11 (1), 781.

8. $\quad$ Slavoff, S. A.; Mitchell, A. J.; Schwaid, A. G.; Cabili, M. N.; Ma, J.; Levin, J. Z.; Karger, A. D.; Budnik, B. A.; Rinn, J. L.; Saghatelian, A., Peptidomic discovery of short open reading frame-encoded peptides in human cells. Nat Chem Biol 2013,9 (1), 59-64. 9. Wilson, B. A.; Foy, S. G.; Neme, R.; Masel, J., Young Genes are Highly Disordered as Predicted by the Preadaptation Hypothesis of De Novo Gene Birth. Nat Ecol Evol 2017, 1 (6), 0146-146.

10. Chu, Q.; Rathore, A.; Diedrich, J. K.; Donaldson, C. J.; Yates, J. R., 3rd; Saghatelian, A., Identification of Microprotein-Protein Interactions via APEX Tagging. Biochemistry 2017, 56 (26), 3299-3306.

11. D'Lima, N. G.; Ma, J.; Winkler, L.; Chu, Q.; Loh, K. H.; Corpuz, E. O.; Budnik, B. A.; Lykke-Andersen, J.; Saghatelian, A.; Slavoff, S. A., A human microprotein that interacts with the mRNA decapping complex. Nat Chem Biol 2017, 13 (2), 174-180.

12. Na, Z.; Luo, Y.; Schofield, J. A.; Smelyansky, S.; Khitun, A.; Muthukumar, S.; Valkov, E.; Simon, M. D.; Slavoff, S. A., The NBDY Microprotein Regulates Cellular RNA Decapping. Biochemistry 2020, 59 (42), 4131-4142.

13. Cao, X.; Khitun, A.; Luo, Y.; Na, Z.; Phoodokmai, T.; Sappakhaw, K.; Olatunji, E.; Uttamapinant, C.; Slavoff, S. A., Alt-RPL36 downregulates the PI3K-AKT-mTOR signaling pathway by interacting with TMEM24. Nat Commun 2021, 12 (1), 508.

14. Chu, Q.; Martinez, T. F.; Novak, S. W.; Donaldson, C. J.; Tan, D.; Vaughan, J. M.; Chang, T.; Diedrich, J. K.; Andrade, L.; Kim, A.; Zhang, T.; Manor, U.; Saghatelian, A., Regulation of the ER stress response by a mitochondrial microprotein. Nat Commun 2019, 10 (1), 4883.

15. King, C.; Sengupta, P.; Seo, A. Y.; Lippincott-Schwartz, J., ER membranes exhibit phase behavior at sites of organelle contact. Proc Natl Acad Sci U S A 2020, 117 (13), 7225-7235.

16. Lafontaine, D. L. J.; Riback, J. A.; Bascetin, R.; Brangwynne, C. P., The nucleolus as a multiphase liquid condensate. Nat Rev Mol Cell Biol 2021, 22 (3), 165-182.

17. Luo, Y.; Na, Z.; Slavoff, S. A., P-Bodies: Composition, Properties, and Functions. Biochemistry 2018, 57 (17), $2424-2431$.

18. Eulalio, A.; Behm-Ansmant, I.; Schweizer, D.; Izaurralde, E., P-body formation is a consequence, not the cause, of RNAmediated gene silencing. Mol Cell Biol 2007, 27 (11), 3970-81.

19. Horvathova, I.; Voigt, F.; Kotrys, A. V.; Zhan, Y.; Artus-Revel, C. G.; Eglinger, J.; Stadler, M. B.; Giorgetti, L.; Chao, J. A., The Dynamics of mRNA Turnover Revealed by Single-Molecule Imaging in Single Cells. Mol Cell 2017, 68 (3), 615-625 e9.

20. Tutucci, E.; Vera, M.; Biswas, J.; Garcia, J.; Parker, R.; Singer, R. H., An improved MS2 system for accurate reporting of the mRNA life cycle. Nat Methods 2018, 15 (1), 81-89.

21. Tibble, R. W.; Depaix, A.; Kowalska, J.; Jemielity, J.; Gross, J. D., Biomolecular condensates amplify mRNA decapping by biasing enzyme conformation. Nat Chem Biol 2021, 17 (5), 615-623.

22. Aumiller, W. M., Jr.; Keating, C. D., Phosphorylation-mediated RNA/peptide complex coacervation as a model for intracellular liquid organelles. Nat Chem 2016, 8 (2), 129-37.

23. Aizer, A.; Kafri, P.; Kalo, A.; Shav-Tal, Y., The P body protein Dcp1a is hyper-phosphorylated during mitosis. PLoS One 2013, 8 (1), e49783.

24. Bearss, J. J.; Padi, S. K.; Singh, N.; Cardo-Vila, M.; Song, J. H.; Mouneimne, G.; Fernandes, N.; Li, Y.; Harter, M. R.; Gard, J. M.; Cress, A. E.; Peti, W.; Nelson, A. D.; Buchan, J. R.; Kraft, A. S.; Okumura, K., EDC3 phosphorylation regulates growth and invasion through controlling P-body formation and dynamics. EMBO Rep 2021, 22 (4), e50835.

25. Banani, S. F.; Lee, H. O.; Hyman, A. A.; Rosen, M. K., Biomolecular condensates: organizers of cellular biochemistry. Nat Rev Mol Cell Biol 2017, 18 (5), 285-298.

26. Alberti, S.; Gladfelter, A.; Mittag, T., Considerations and Challenges in Studying Liquid-Liquid Phase Separation and Biomolecular Condensates. Cell 2019, 176 (3), 419-434.

27. Sanders, D. W.; Kedersha, N.; Lee, D. S. W.; Strom, A. R.; Drake, V.; Riback, J. A.; Bracha, D.; Eeftens, J. M.; Iwanicki, A.; Wang A.; Wei, M. T.; Whitney, G.; Lyons, S. M.; Anderson, P.; Jacobs, W. M.; Ivanov, P.; Brangwynne, C. P., Competing Protein-RNA Interaction Networks Control Multiphase Intracellular Organization. Cell 2020, 181 (2), 306-324.e28.

28. Yang, P.; Mathieu, C.; Kolaitis, R. M.; Zhang, P.; Messing, J.; Yurtsever, U.; Yang, Z.; Wu, J.; Li, Y.; Pan, Q.; Yu, J.; Martin, E. W.; Mittag, T.; Kim, H. J.; Taylor, J. P., G3BP1 Is a Tunable Switch that Triggers Phase Separation to Assemble Stress Granules. Cell 2020, 181 (2), 325-345.e28. 
29. Guillen-Boixet, J.; Kopach, A.; Holehouse, A. S.; Wittmann, S.; Jahnel, M.; Schlussler, R.; Kim, K.; Trussina, I.; Wang, J.; Mateju, D.; Poser, I.; Maharana, S.; Ruer-Gruss, M.; Richter, D.; Zhang, X.; Chang, Y. T.; Guck, J.; Honigmann, A.; Mahamid, J.; Hyman, A. A.; Pappu, R. V.; Alberti, S.; Franzmann, T. M., RNA-Induced Conformational Switching and Clustering of G3BP Drive Stress Granule Assembly by Condensation. Cell 2020, 181 (2), 346-361 e17.

30. Fromm, S. A.; Kamenz, J.; Noldeke, E. R.; Neu, A.; Zocher, G.; Sprangers, R., In vitro reconstitution of a cellular phasetransition process that involves the mRNA decapping machinery. Angew Chem Int Ed Eng/ 2014, 53 (28), 7354-9.

31. Schutz, S.; Noldeke, E. R.; Sprangers, R., A synergistic network of interactions promotes the formation of in vitro processing bodies and protects mRNA against decapping. Nucleic Acids Res 2017, 45 (11), 6911-6922.

32. Burke, K. A.; Janke, A. M.; Rhine, C. L.; Fawzi, N. L., Residue-by-Residue View of In Vitro FUS Granules that Bind the CTerminal Domain of RNA Polymerase II. Mol Cell 2015, 60 (2), 231-41.

33. Hubstenberger, A.; Courel, M.; Benard, M.; Souquere, S.; Ernoult-Lange, M.; Chouaib, R.; Yi, Z.; Morlot, J. B.; Munier, A.; Fradet, M.; Daunesse, M.; Bertrand, E.; Pierron, G.; Mozziconacci, J.; Kress, M.; Weil, D., P-Body Purification Reveals the Condensation of Repressed mRNA Regulons. Mol Cell 2017, 68 (1), 144-157 e5.

34. Sharma, K.; D'Souza, R. C.; Tyanova, S.; Schaab, C.; Wisniewski, J. R.; Cox, J.; Mann, M., Ultradeep human phosphoproteome reveals a distinct regulatory nature of Tyr and Ser/Thr-based signaling. Cell Rep 2014, 8 (5), $1583-94$.

35. Nigg, E. A., Mitotic kinases as regulators of cell division and its checkpoints. Nat Rev Mol Cell Biol 2001, 2 (1), $21-32$.

36. Asghar, U.; Witkiewicz, A. K.; Turner, N. C.; Knudsen, E. S., The history and future of targeting cyclin-dependent kinases in cancer therapy. Nat Rev Drug Discov 2015, 14 (2), 130-46.

37. Luo, Y.; Schofield, J. A.; Na, Z.; Hann, T.; Simon, M. D.; Slavoff, S. A., Discovery of cellular substrates of human RNA-decapping enzyme DCP2 using a stapled bicyclic peptide inhibitor. Cell Chem Biol 2020.

38. Kiyatkin, A.; Aksamitiene, E.; Markevich, N. I.; Borisov, N. M.; Hoek, J. B.; Kholodenko, B. N., Scaffolding protein Grb2associated binder 1 sustains epidermal growth factor-induced mitogenic and survival signaling by multiple positive feedback loops. $J$ Biol Chem 2006, 281 (29), 19925-38.

39. Zhao, B. X.; Wang, J.; Song, B.; Wei, H.; Lv, W. P.; Tian, L. M.; Li, M.; Lv, S., Establishment and biological characteristics of acquired gefitinib resistance in cell line $\mathrm{NCl}-\mathrm{H} 1975 /$ gefinitib-resistant with epidermal growth factor receptor T790M mutation. $\mathrm{Mol}$ Med Rep 2015, 11 (4), 2767-74.

40. Wee, P.; Wang, Z., Epidermal Growth Factor Receptor Cell Proliferation Signaling Pathways. Cancers (Basel) 2017, 9 (5).

41. Fan, Q. W.; Cheng, C.; Knight, Z. A.; Haas-Kogan, D.; Stokoe, D.; James, C. D.; McCormick, F.; Shokat, K. M.; Weiss, W. A., EGFR signals to mTOR through PKC and independently of Akt in glioma. Sci Signal 2009, 2 (55), ra4.

42. Van Treeck, B.; Protter, D. S. W.; Matheny, T.; Khong, A.; Link, C. D.; Parker, R., RNA self-assembly contributes to stress granule formation and defining the stress granule transcriptome. Proc Natl Acad Sci U S A 2018, 115 (11), $2734-2739$.

43. Rai, A. K.; Chen, J. X.; Selbach, M.; Pelkmans, L., Kinase-controlled phase transition of membraneless organelles in mitosis. Nature 2018, 559 (7713), 211-216.

44. Nissan, T.; Parker, R., Analyzing P-bodies in Saccharomyces cerevisiae. Methods Enzymol 2008, 448, 507-20.

45. Pervushin, K.; Riek, R.; Wider, G.; Wuthrich, K., Attenuated T2 relaxation by mutual cancellation of dipole-dipole coupling and chemical shift anisotropy indicates an avenue to NMR structures of very large biological macromolecules in solution. Proc Natl Acad Sci U S A 1997, 94 (23), 12366-71.

46. Delaglio, F.; Grzesiek, S.; Vuister, G. W.; Zhu, G.; Pfeifer, J.; Bax, A., NMRPipe: a multidimensional spectral processing system based on UNIX pipes. J Biomol NMR 1995, 6 (3), 277-93.

47. Tiscornia, G.; Singer, O.; Verma, I. M., Production and purification of lentiviral vectors. Nat Protoc 2006, 1 (1), $241-5$.

48. $\quad$ Castaneda-Bueno, M.; Arroyo, J. P.; Zhang, J.; Puthumana, J.; Yarborough, O., 3rd; Shibata, S.; Rojas-Vega, L.; Gamba, G.; Rinehart, J.; Lifton, R. P., Phosphorylation by PKC and PKA regulate the kinase activity and downstream signaling of WNK4. Proc Natl Acad Sci U S A 2017, 114 (5), E879-E886. 


\section{TABLE OF CONTENT GRAPHIC}

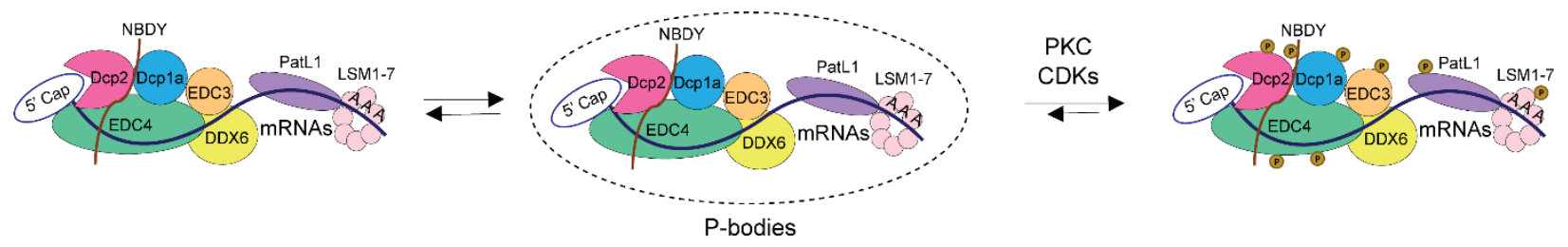

